\title{
Apropriação das Tecnologias da Informação e Comunicação no ensino de ciências: uma revisão sistemática da última década (2007-2016)
}

\author{
Rafaela da Silva Reis, PPGEC, UFRPE - rafaelaaa.reis@ hotmail.com \\ Bruno Silva Leite, UAST, UFRPE - leitebrunosilva@gmail.com \\ Marcelo Brito Carneiro Leão, PPGEC, UFRPE - mbcleao@terra.com.br
}

Resumo: Este trabalho tem como propósito analisar o objetivo das pesquisas envolvendo as Tecnologias da Informação e Comunicação (TIC) no Ensino de Ciências. Para isso, realizou-se uma revisão sistemática em 11 revistas da área de Ensino no período de 2007-2016. Nesse sentido, a partir de três critérios pré-estabelecidos investigamos nos artigos a intencionalidade da pesquisa em analisar as estratégias de uso das TIC, as contribuições e limitações do uso das TIC e o interesse na aplicação/utilização das TIC. Os resultados mostram que dos 291 artigos analisados, 47\% são direcionados para a utilização de algum material suportado pelas TIC, sem a preocupação de quem (o professor) irá utilizar ou como (a estratégia) será essa utilização. Além disso, identificamos a necessidade de pesquisas voltadas à elaboração de estratégias para o uso adequado das TIC.

Palavras-chave: TIC, Ensino de Ciências, Revisão Sistemática.

\section{Appropriation of Information and Communication Technologies in science education: a systematic review of the last decade (2007-2016)}

\begin{abstract}
This paper proposes analyses the objective of research involving Information and Communication Technologies (ICT) in Science Teaching. For this, a systematic review was carried out in 11 journals of the area of Education in the period 2007-2016. In this sense, based on three pre-established criteria, we investigated in the articles the intentionality of the research in analyzing the strategies of use of ICT, the contributions and limitations of the use of ICT, and the interest in the application of ICT. The results show that of the 291 articles analyzed, $47 \%$ is directed towards the use of some material supported by ICT, without the preoccupation of who (the Teacher) will use it or how (the strategy) it will be used. Furthermore, we identified the need for research aimed at developing strategies for the appropriate use of ICT.
\end{abstract}

Keywords: ICT, Science teaching, Systematic review.

\section{Introdução}

Ao longo dos anos, as fronteiras entre educação e tecnologias têm se estreitado cada vez mais. Por onde quer que olhemos, a tecnologia está presente. Na sala de aula não é diferente. Seja com o professor ou com o aluno, as tecnologias têm contribuído para a construção do conhecimento. Pesquisas conduzidas no Brasil mostram que as tecnologias ganham cada vez mais espaço e estão muito próximas de grande parte da população, embora ainda sejam encontradas disparidades no acesso e no uso da rede, sobretudo quando verificamos os dados sobre a banda larga nos domicílios de baixa renda (Barbosa, 2016).

O uso das tecnologias tem possibilitado o acesso a uma educação, talvez, diferenciada considerando que sua inserção nas escolas é um fenômeno em franca expansão. Conhecer a real capacidade que as tecnologias têm para contribuir com o processo de ensino e aprendizagem é um passo fundamental. No ensino de ciências, por exemplo, ela pode ter um papel essencial de auxiliar o professor na construção do 
conhecimento de seus alunos, na aprendizagem dos conteúdos científicos destes e na minimização das limitações encontradas durante sua formação.

É importante percebermos a necessidade da escola em se apropriar das tecnologias da informação e comunicação (TIC), integrando-as ao processo de ensino e aprendizagem através de seus protagonistas, alunos e professores, reforçando seu compromisso na formação de cidadãos conscientes do seu papel transformador numa sociedade mais justa e igualitária (Leão, 2011). Essa necessidade é observada no sentido de que as TIC têm se mostrado como um instrumento que pode facilitar a compreensão dos conceitos na área de ensino das ciências, tornando-os menos abstratos, mais "visualizáveis" e compreensíveis do que são considerados pelos estudantes. Através da tecnologia é possível a realização de práticas pedagógicas diversificadas, a exemplo do uso de um jogo eletrônico, que, segundo Ignácio (2013), promove com a sua realização, um aprendizado que ultrapassa os conteúdos químicos presentes num currículo escolar, além de acontecer de forma motivadora e prazerosa. Acreditamos que o aumento do uso e da apropriação das TIC em grandes setores da população tem provocado importantes mudanças sociais nos últimos anos. Leite (2016) destaca que o interesse na aplicação das tecnologias em atividades relacionadas com o ensino e aprendizagem tem conduzido a uma ruptura das metodologias tradicionais de ensino que estão "cristalizadas" com o tempo.

A literatura tem destacado que as tecnologias são propostas com o intuito de melhorar o desempenho dos estudantes frente aos conteúdos escolares, tornando esses assuntos menos abstratos, não minimizando o papel fundamental dos professores no processo de ensino e aprendizagem (Pretto; Riccio, 2010; Coll; Monereo, 2010; Leão, 2011; Leite, 2015; Rosa; Eichler; Catelli, 2015; Martín, 2017). Contudo, precisamos enfatizar que a inserção das tecnologias nas escolas não deve ser vista como uma solução para todos os problemas da educação, mas, como um recurso que deve ser somado ao processo de ensino e aprendizagem. Ainda nesse sentido, Leão (2011) enfatiza que a utilização das TIC na educação pode gerar uma expectativa, talvez exagerada, de que estes ambientes garantirão uma excelência na aprendizagem. $\mathrm{O}$ autor acredita que a mera "transfiguração" de uma roupagem antiga para a utilização de recursos tecnológicos de ponta não trará grandes mudanças. Para o sucesso desta incorporação, a utilização das TIC na educação terá que vir acompanhada de uma profunda discussão e análise das estratégias metodológicas, que possam ajudar na construção de uma aprendizagem significativa para o aluno.

Durante as últimas décadas as tecnologias têm sido inseridas nas práticas docentes, tanto na educação básica como na superior, sendo relatadas em diversos periódicos especializados. Nesse sentido, nossa pesquisa (parte dos resultados da dissertação de mestrado) busca analisar qual tem sido o foco das pesquisas em torno do uso das TIC no ensino de ciências. Na qual questionamos:

$\checkmark$ O foco das pesquisas publicadas nos periódicos está na simples utilização de um material digital ou está em como o material digital tem sido utilizado?

$\checkmark$ Há uma preocupação da comunidade científica em analisar como as tecnologias têm sido utilizadas em sala de aula?

No intuito de alcançar essas respostas, o mapeamento dar-se-á por meio de uma revisão sistemática de literatura.

Dessa maneira, este artigo foi divido em quatro seções: a primeira seção apresenta uma breve contextualização sobre as TIC no ensino; a segunda seção, descreve os procedimentos metodológicos aplicados para a coleta e análise dos dados neste estudo; na terceira seção, apresenta os resultados das análises e discussões 
alcançados durante a pesquisa; na quarta e última seção, dedica-se as considerações finais acerca da temática da pesquisa e trabalhos futuros.

\section{Metodologia}

Para contemplar os objetivos da pesquisa, realizamos uma revisão sistemática da literatura para analisar qual tem sido o foco das pesquisas em torno do uso das TIC no Ensino de Ciências. Segundo Sampaio e Mancini (2007, p. 84), a revisão sistemática de literatura "é um tipo de estudo retrospectivo e secundário", que utiliza a literatura sobre determinado tema como fonte primária de dados, a fim de obter um resumo de evidências, mediante a sistematização e aplicação de métodos explícitos de busca, apreciação crítica e síntese de informação selecionada. Kitchenham (2004) destaca que esta revisão objetiva identificar, avaliar e interpretar as pesquisas relevantes sobre determinada temática, a fim de responder um questionamento de pesquisa bem delimitado. A revisão sistemática leva em consideração o crescimento da informação científica, pois ajuda a sintetizar a evidência disponível na literatura sobre uma intervenção, e assim auxiliar o trabalho de profissionais e pesquisadores (Sampaio; Mancini, 2007). As etapas adotadas nesta revisão sistemática foram: definição do objetivo, da pergunta de pesquisa, de banco de dados, dos critérios de inclusão e exclusão, das palavras-chave; seleção de trabalhos; análise e síntese dos estudos incluídos na revisão sistemática de literatura; redação; publicação dos resultados (Kitchenham, 2004; Sampaio; Mancini, 2007; De-La-Torre-Ugarte-Guanilo; Takahashi; Bertolozzi, 2011; Galvão; Pereira, 2014; Coelho Neto; Blanco; Silva, 2017).

Para a coleta de dados selecionamos artigos de 11 periódicos (nacionais e internacionais). $\mathrm{O}$ critério para escolha destes periódicos pautou-se nos estratos indicativos de qualidade (Qualis-periódicos A1 e A2), conforme quadriênio 2013-2016 da plataforma sucupira e que são referenciados por boa parte da comunidade acadêmica como os mais importantes na área de ensino de ciências, além de conterem, em uma primeira análise, artigos na área das TIC no ensino de ciências. Os periódicos foram: 1) Revista Brasileira de Pesquisa em Educação em Ciências (RBPEC), 2) Investigações em Ensino de Ciências (IENCI), 3) Ciência e Educação, 4) Revista Ensaio Pesquisa em Educação em Ciências (ENSAIO), 5) Enseñanza de las Ciencias, 6) Revista Brasileira de Ensino de Ciência e Tecnologia (RBECT), 7) Alexandria: Revista de Educação em Ciência e Tecnologia (ALEXANDRIA), 8) Revista de Ensino de Ciências e Matemática (RenCiMa), 9) Revista Electrónica de Investigación en Educación en Ciencias (REIEC), 10) Revista Latino-americana de Tecnologia Educativa, 11) Educación Química. De maneira a contribuir para a fluidez na leitura do texto, atribuímos aos periódicos os códigos R1, R2, R3, ..., R11, respectivamente.

Para análise dos dados, o período escolhido foi das publicações dos últimos 10 anos (2007 a 2016). Os critérios de inclusão para a escolha das produções foram:

i. Critério 1 (C1): A intencionalidade da pesquisa em analisar o uso das TIC pelo professor no ensino de ciências (interesse pelas estratégias de uso das TIC);

ii. Critério 2 (C2): contribuições e limitações do uso das TIC no ensino (interesse pela funcionalidade das TIC);

iii. Critério 3 (C3): somente aplicação/utilização de tecnologias no ensino (interesse no uso das TIC).

Além dos critérios acima descritos, durante o levantamento e seleção dos artigos tivemos como orientação uma série de palavras-chave correlatas ao tema, algumas são: TIC, Tecnologia, Digital, Recurso didático digital, Computador, Software, informática, Web, virtual, além de suas variações no plural. Buscamos também o cruzamento dessas 
palavras-chave com os termos ensino/educação, ciências e Ensino de Ciências. Após a seleção dos artigos foi realizada a leitura dos seus resumos, considerando que estes apresentam uma boa forma de identificar os objetivos do trabalho. Quando essa informação não estava explícita no resumo, se fez necessário a leitura também na íntegra, possibilitando verificar se os artigos se encaixavam em alguns dos critérios estabelecidos. Assim, na análise de cada volume/edição dos periódicos no último decênio foram selecionados 352 artigos, que após o refinamento durante a leitura dos resumos e texto completo, totalizaram 291 trabalhos envolvendo o uso das TIC no ensino de ciências. $\mathrm{Na}$ análise e síntese dos dados foram extraídos trechos de alguns artigos que responderam às perguntas de pesquisa desta revisão sistemática. Na seção 3 esses resultados são expostos e discutidos.

\section{Resultados e discussões}

Esta seção está dividida em duas partes, na primeira destacamos os resultados observados a partir dos trabalhos primários selecionados; na segunda descrevemos as análises realizadas a partir dos dados encontrados nos trabalhos primários selecionados.

\subsection{Descrição geral dos trabalhos primários analisados}

Os resultados obtidos em nossa pesquisa permitiram identificar um perfil dos artigos estudados. Realizamos uma análise com relação à quantidade de produção das pesquisas ao longo do tempo. A partir dos 352 trabalhos primários pré-selecionados encontrados na análise das plataformas dos periódicos, 291 artigos científicos apresentavam os dados necessários para a pesquisa.

Observamos que o uso das TIC, embora seja amplamente discutido nas universidades e encontros científicos, é relativamente pouco estudado quando comparado ao número de pesquisas publicadas nas 11 revistas investigadas (Gráfico 1). Todavia, é observado um aumento significativo no número de pesquisas sobre o uso das TIC ao longo dos últimos dez anos (período analisado), quando comparamos 2007 (13 publicações sobre as TIC) e 2016 (41 publicações).

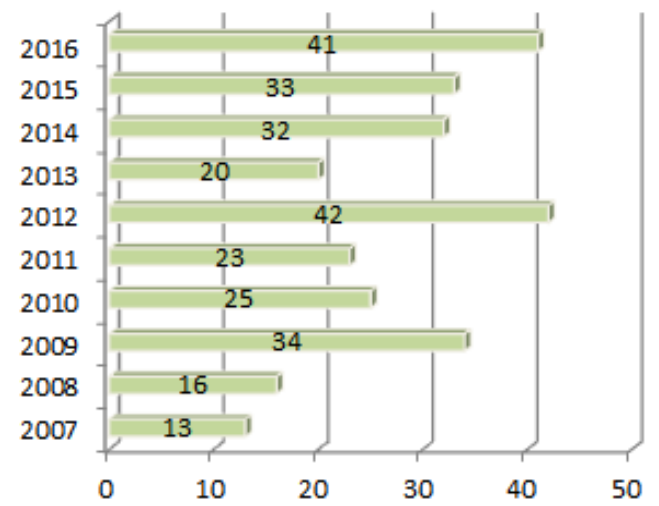

Gráfico 1 - Número de publicações selecionadas por ano analisado.

Observando os resultados no Gráfico 1, podemos conjecturar que o número de publicações tem crescido, em geral, a cada três anos, tendo um decréscimo no ano seguinte e posteriormente retornando seu crescimento. Nessa observação, temos como exceção o ano de 2016, o que evidencia a importância de se investigar a próxima 
sequência de três anos (2016, 2017 e 2018) e verificar se ocorre o aumento periódico ou se foi apenas uma "coincidência" nos resultados.

$\mathrm{Na}$ leitura dos artigos buscamos identificar se havia a presença de algum dos três critérios: C1, C2 e C3. Destacamos que durante toda a pesquisa não encontramos qualquer revisão sistemática de literatura ou similar sobre o uso das TIC (dentro de nossos objetivos), o que enaltece o caráter inédito e investigativo desta pesquisa.

Dentre os 291 artigos selecionados, a distribuição entre os critérios se deu conforme o Gráfico 2.

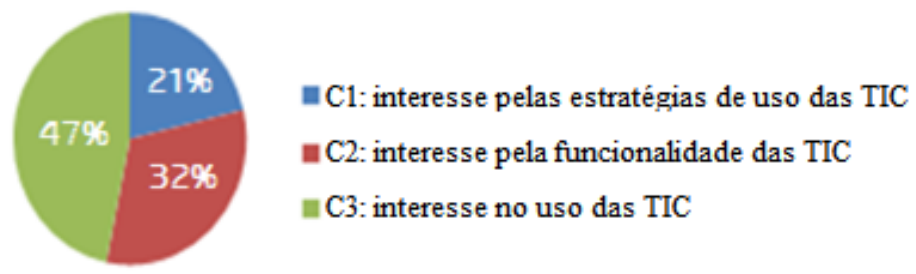

Gráfico 2 - Percentual do número de artigos classificados em cada critério.

Esses dados, inicialmente, evidenciam nossa hipótese primária de que a preocupação em torno das estratégias de uso das TIC (21\%) é pouca (menos da metade) em relação às pesquisas de utilização/aplicação das TIC (47\%) no ensino de ciências. Isso corrobora com as ideias de Leão (2011) destacando que a utilização das TIC na educação está gerando uma expectativa exagerada, em alguns casos, de que ela irá garantir uma excelência na aprendizagem. Se essa utilização não vier acompanhada de uma análise das estratégias em torno do seu uso, acreditamos que não haverá grandes mudanças. É importante que os resultados observados em $\mathrm{C} 1$, motivem os pesquisadores/professores a mudarem este quadro, e passem a considerar como necessário a implementação de estratégias no uso das TIC no ensino de ciências. Os dados de C2 (32\%) buscam analisar as contribuições e limitações de uso das TIC, demostrando preocupação com o recurso utilizado.

Encontra-se no Gráfico 3 a sistematização das informações obtidas durante nossa pesquisa, apresentando um panorama de como está distribuído os critérios em cada revista.

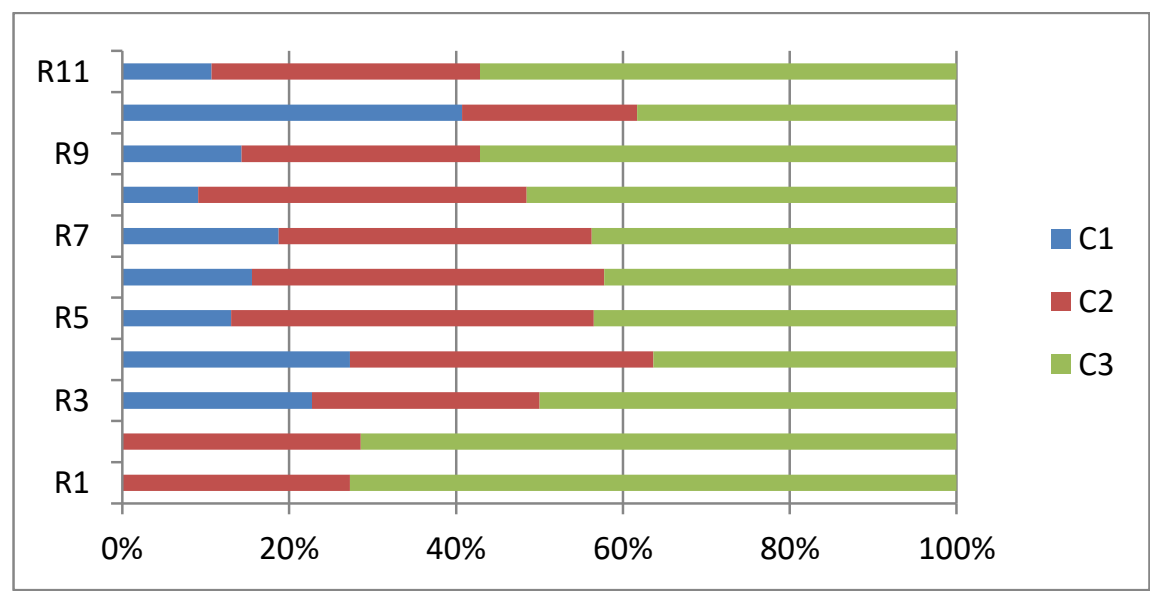

Gráfico 3 - Distribuição dos critérios em cada revista analisada.

Com base no Gráfico 3, destacamos que a revista R10 tem maior porcentagem de artigos utilizando o critério $\mathrm{C} 1$, demonstrando uma possível preocupação com as publicações acerca das estratégias de uso das tecnologias na educação. Em contra 
partida, as revistas R1 e R2 não apresentam, em um período de 10 anos, nenhuma publicação com as características de classificação em C1. Embora sejam revistas conceituadas na área de ensino de ciências, tal lacuna demonstra a necessidade de pesquisas que direcionem o uso das TIC por meio de estratégias. Como já evidenciado no Gráfico 2, o critério 3 é o que apresenta maior quantidade de trabalhos relacionados a este tema, ou seja, pouca importância se tem dado a incorporação das TIC com uso de estratégias, considerando apenas o uso da tecnologia.

\subsection{Informações extraídas com a análise dos trabalhos primários}

Ao analisar os trabalhos primários através da leitura na íntegra de seu conteúdo, Silva e Brito (2014) afirmam que algumas características podem ser extraídas a fim de auxiliar o desenvolvimento de atividades de pesquisa posteriores. Assim, nosso intuito não foi apenas de classificar as pesquisas nos critérios abordados inicialmente, mas também estabelecermos entre os critérios uma ordem de importância, baseado no que foi discutido anteriormente.

Entendemos que a mera utilização das TIC na educação não trará os resultados que esperam as pesquisas da área se elas forem usadas de forma redutiva e inadequada pelo professor. Para o sucesso de sua utilização, ela deve vir acompanhada de uma profunda discussão e análise das estratégias metodológicas, que possam ajudar na construção de uma aprendizagem significativa para o aluno (Leão, 2011; Leite, 2015). Neste sentido, estabelecemos o critério $\mathrm{C} 1$ como o critério mais importante frente aos outros critérios, pois ele contempla pesquisas que tem a preocupação na análise de estratégias em torno do uso das TIC pelo professor. Como segundo critério mais importante sugerimos o $\mathrm{C} 2$, devido à pesquisa ter $\mathrm{o}$ interesse de analisar a funcionalidade das TIC, percebendo em que deve ser melhorada, analisando a utilidade e aceitabilidade pelos alunos. Por último, classificamos o C3 como de menor importância, tendo em vista o que já colocamos anteriormente sobre a mera "transfiguração" de uma roupagem antiga para a utilização de recursos tecnológicos.

Definido a ordem de importância aos critérios estabelecidos, contabilizamos a quantidade que cada um é observado por ano (Tabela 1).

Tabela 1 - Número de artigos publicados por ano relacionados com os Critérios.

\begin{tabular}{|c|c|c|c|c|c|c|c|c|c|c|}
\hline Ano/critério & $\mathbf{2 0 0 7}$ & $\mathbf{2 0 0 8}$ & $\mathbf{2 0 0 9}$ & $\mathbf{2 0 1 0}$ & $\mathbf{2 0 1 1}$ & $\mathbf{2 0 1 2}$ & $\mathbf{2 0 1 3}$ & $\mathbf{2 0 1 4}$ & $\mathbf{2 0 1 5}$ & $\mathbf{2 0 1 6}$ \\
\hline C1 & 4 & 3 & 9 & 4 & 6 & 6 & 3 & 5 & 11 & 10 \\
\hline C2 & 2 & 4 & 11 & 7 & 9 & 10 & 7 & 10 & 10 & 15 \\
\hline C3 & 7 & 9 & 14 & 14 & 8 & 26 & 10 & 17 & 12 & 16 \\
\hline
\end{tabular}

A partir dos dados obtidos em nossa investigação, observamos que a quantidade de trabalhos definida no critério 3 é maior que a quantidade definida no critério 2 e esse maior que no critério 1, ou seja, os artigos analisados na pesquisa em torno do uso das TIC demostram estar mais voltados à utilização de um recurso tecnológico do que como se deve utilizar esse recurso (que estratégias deve ser utilizadas) no processo de ensino e aprendizagem. Enquanto a ordem de importância se dá do C1 para o C3, a quantidade se dá inversamente, ou seja, do C3 para o $\mathrm{C} 1$. Ademais, algumas revistas, como observamos no gráfico 3, não publicaram nenhum artigo com o critério 1 , ou seja, nenhum artigo publicado no período de 10 anos que indicasse a preocupação com as estratégias em torno da utilização das TIC na educação. Essa constatação confirma nossa preocupação em torno do uso das TIC pelos professores. Acreditamos que na aplicação de um recurso não há oportunidades de aprendizagem sem a mediação do professor, sem uma estratégia planejada. Como afirma Demo (2005, p.12) "qualquer 
artefato técnico implantado na escola só frutifica sob a mediação do professor". Eichler e Del Pino (2000) destacam que as TIC por si só não resolvem o problema de aprendizagem do estudante, haja visto que é necessário que essas tecnologias estejam em sintonia com as diretrizes legislativas da escola e com as possibilidades pedagógicas do professor. Ainda em relação aos dados da Tabela 1 podemos identificar que o número de pesquisas em C1 em 2016 é bem maior que no início em 2007, assim também em C2 e C3, porém, neste (C3), outros anos foram bem maiores, como em 2012 com 26 artigos publicados.

A seguir destacamos resumidamente alguns artigos que foram categorizados em nossa pesquisa pertencentes ao critério 1 (C1). Em 2015, ano com a maior quantidade de artigos publicados com esse critério (11 no total), o artigo da R4, intitulado "Quem me salva de TI?": representações docentes sobre a tecnologia digital (Rosa; Eichler; Catelli, 2015) investiga as representações dos docentes sobre a relação entre o ensino dos conteúdos programáticos da disciplina de química e as tecnologias digitais, com a finalidade de identificar aspectos que permeiam e/ou circundam as estratégias didáticopedagógicas. Constatou-se a existência de divergências com relação aos discursos e às práticas no que concerne à utilização das tecnologias digitais e, devido à preocupação quanto sua utilização, esse artigo foi enquadrado nesse critério. O trabalho Estudo das Funções Afins, Quadráticas e Equações Polinomiais com o auxílio do software Winplot no Ensino Médio (Jesus; Silva, 2015), publicado na R6, busca analisar através de relatos e discussões as dificuldades que os professores de matemática ainda encontram em introduzir em suas atividades de ensino o uso de recursos tecnológicos. Em 2014, o artigo da R9 intitulado de Atividades experimentais e atividades baseadas em simulações computacionais: quais os principais fatores que influenciam a decisão de professores de conduzir ou não essas práticas em suas aulas? (Heidemann; Araujo; Veit, 2014) analisa os principais fatores que influenciam na decisão de professores de Física de usar ou não atividades experimentais e atividades com simulações computacionais como estratégias de ensino. É interessante destacar a preocupação dos autores em descrever os fatores geradores da decisão dos professores de usar ou não outros recursos em sala de aula, demonstrando que usar uma ferramenta de ensino não é tão óbvio e de escolha certa para todos os professores. Esses artigos demostram a preocupação dos seus autores em analisar a inserção das TIC baseados no uso de estratégias, assim sendo classificados em $\mathrm{C} 1$.

Em relação ao critério 2 (C2) destacamos o artigo que a R1 publicou intitulado de Fundamentos Pedagógicos para o Uso de Simulações e Laboratórios Virtuais no Ensino de Ciências (Paula, 2017). Esse trabalho interpreta possíveis contribuições dos aplicativos de computador que contêm animações, simulações e laboratórios virtuais a partir de uma perspectiva sócio histórica do ensino e da aprendizagem. De maneira geral ele visa identificar as potencialidades e as limitações desses aplicativos. Outro artigo que podemos também fazer referência é o da R6, Contribuição da Web 2.0 como ferramenta de aprendizagem: um estudo de caso (Leite; Leão, 2015). Esse artigo tem o objetivo de analisar o papel de algumas ferramentas da Web 2.0 e como essas poderiam contribuir para uma aprendizagem ampla e flexível por parte dos usuários. $\mathrm{Na} \mathrm{R4,}$ podemos destacar o artigo O computador no ensino de química: Impressões versus realidade. Em Foco as escolas públicas da Baixada Fluminense (Benite; Benite, 2008) com o objetivo de refletir em bases sócio-histórica e culturais, sobre a compreensão das potencialidades e limitações do uso do computador em escolas do ensino médio da rede pública da Baixada Fluminense, para o ensino de química. Esses artigos demonstram a preocupação dos seus autores em analisar a funcionalidade, contribuições/limitações das TIC, conforme classificamos o critério 2. 
Por último, em relação ao critério 3 (C3) o artigo Um estudo exploratório para avaliar a dificuldade de problemas em ensino de Física utilizando a teoria da carga cognitiva com o auxílio de uma hipermídia (Oliveira; Moreira, 2016) publicado na R6, apresenta um estudo exploratório sobre a resolução de problemas com o auxílio de uma hipermídia. Na mesma revista temos o artigo Ensinando e Aprendendo Geometria Plana Através de Vídeo Educativo: Algumas Sugestões de Atividades Didáticas para Aulas de Matemática no Ensino Médio (Santos, 2014) com o objetivo de propor a utilização de vídeos educativos como atividade didática para os professores de matemática do Ensino Médio na abordagem de conteúdos de geometria plana a partir da exibição do filme "Nas malhas da geometria", propondo o desenvolvimento de trabalhos pedagógicos interdisciplinares, visando a construção do pensamento geométrico. Na R10, temos o artigo El uso de la geolocalización en educación secundaria para la mejora del aprendizaje situado (Gros; Forés, 2013) que descreve duas experiências de aprendizagem situada que integra aplicativos móveis e ferramentas de geolocalização em projetos educacionais de salas de aula do ensino médio. Esses artigos demostram que seus autores fizeram uso das TIC, porém sem investigar as estratégias ou contribuições/limitações do recurso, assim sendo classificados em C3.

Dos 352 artigos inicialmente analisados, 291 artigos foram classificados em um dos três critérios pré-estabelecidos. Os 61 artigos restantes foram excluídos após o refinamento durante a análise dos resumos e do texto completo, pois não foi observado, durante a leitura, nenhum dos critérios propostos. A título de exemplo, destacamos alguns desses artigos. Em 2011, percebendo a escassez de pesquisadores desenvolvendo pesquisas nesse campo, a R7 fez uma chamada para os artigos com a temática "Formação de professores em ciências e tecnologia" em virtude da constatação de uma grande demanda sobre esse tema na área e uma carência de pesquisadores que desenvolvem suas pesquisas nesse campo. Na edição de 2012, n. 2, saíram os trabalhos advindos da chamada de artigos para a edição temática. Foram publicados artigos sobre "Trajetórias formativas docentes", "Discurso pedagógico", "Textos de divulgação científica", "História da ciência através do cinema", "Saberes docentes", entre outros. Nenhum desses artigos destacou os critérios considerados nessa pesquisa. Na R6 o artigo $O$ arborescer das TIC na Educação: da raiz aos ramos mais recentes (Ataíde; Mesquita, 2014), apresentou uma revisão bibliográfica propondo descrever a trajetória histórica das TIC na educação, mostrando que o progresso observado previa (seguia na direção de) uma introdução maciça de tecnologias no ambiente escolar. Esses artigos não atenderam aos critérios estabelecidos $(\mathrm{C} 1, \mathrm{C} 2$ ou $\mathrm{C} 3)$ por seus objetivos se tratarem de fazer uma chamada de artigos e realizar uma revisão bibliográfica, respectivamente, não de avaliar estratégias, funcionalidades ou utilização prática de algum recurso tecnológico.

Ao final desta etapa de nossa pesquisa, destacamos que as tecnologias estão cada vez mais presentes na sala de aula e que o desenvolvimento e discussões sobre estratégias que possibilitem ao professor se apropriar das tecnologias em sua prática docente, é extremamente importante.

\section{Conclusões}

Esta pesquisa visou apresentar uma revisão sistemática da literatura sobre as TIC no ensino de ciências buscando responder aos seguintes questionamentos: $\mathrm{O}$ foco das pesquisas publicadas nos periódicos está na simples utilização de um material digital ou está em como o material digital tem sido utilizado? Há uma preocupação da comunidade científica em analisar como as tecnologias têm sido utilizadas em sala de 
aula? Ao fazer o levantamento das publicações aqui proposto, analisamos 291 artigos classificando-os em três critérios de apropriação: interesse pelas estratégias de uso das TIC (C1), interesse pela funcionalidade das TIC (C2) e interesse no uso das TIC (C3).

Em relação ao primeiro questionamento, analisando as publicações ano a ano, podemos identificar que em 2016 o número de publicações é maior do que em $2007 \mathrm{em}$ todos os critérios, indicando o interesse crescente das pesquisas em torno das TIC no ensino de ciências. Ressaltamos que dos 291 artigos analisados, 21\% (62) foram classificados como C1, 32\% (93) como C2 e 47\% (136) como C3. Dessa forma, os dados permitem inferir que o foco maior das publicações nos últimos dez anos, nas onze revistas investigadas (de alto estrato de avaliação - Qualis A1 e A2) está na utilização de algum material suportado pelas TIC, sem a preocupação de quem (o professor) irá utilizar ou como (a estratégia) se dá essa utilização. Baseados nesses dados, podemos observar que a comunidade científica tem pouco investigado sobre o uso das TIC em sala de aula. Nesse contexto, identificamos a necessidade de pesquisas em analisar as estratégias em torno de seu uso, almejando que com os resultados desta pesquisa, outros pesquisadores de diversas áreas (não só de ensino das ciências) percebam essa lacuna e busquem saná-la com a análise e elaboração de estratégias para o uso adequado das TIC.

Por fim, gostaríamos de reafirmar que o professor tem um papel fundamental na utilização das TIC e, se esse uso for feito de modo despreocupado e simplista, não trará grandes mudanças. Relembrando a afirmação de Demo (2005, p.12) "parece evidente a dificuldade de transformar as tecnologias em oportunidades de aprendizagem sem a mediação do professor. Qualquer artefato técnico implantado na escola só frutifica sob a mediação do professor".

\section{Agradecimentos}

A Coordenação de Aperfeiçoamento de Pessoal de Nível Superior (CAPES).

\section{Referências}

ATAÍDE, J. F.; MESQUITA, N. A. S. O Arborescer das TIC na Educação: da raiz aos ramos mais recentes. Revista Brasileira de Ensino de Ciência e Tecnologia, v. 7, n. 1, p. 82-106, 2014.

BARBOSA, A. F. Pesquisa sobre o uso das tecnologias de informação e comunicação nas escolas brasileiras: TIC educação 2015, São Paulo: Comitê Gestor da Internet no Brasil, 2016.

BENITE, A. M. C.; BENITE, C. R. M. O computador no ensino de química: Impressões versus Realidade. Em Foco as Escolas Públicas da Baixada Fluminense. Ensaio Pesquisa em Educação em Ciências, v. 10, n. 2, p.320-339, 2008.

COELHO NETO, J.; BLANCO, M. B.; SILVA, J. A. O uso de gamificação e dificuldades matemáticas: possíveis aproximações. RENOTE, v. 15, n. 1, 2017.

COLL, C; MONEREO, C. Psicologia da Educação Virtual: Aprender e ensinas com as tecnologias da informação e da comunicação. Artmed Editora, 2010.

DE-LA-TORRE-UGARTE-GUANILO, M. C.; TAKAHASHI, R. F; BERTOLOZZI, M. R. Revisão sistemática: noções gerais. Revista da Escola de Enfermagem da USP, v. 45, n. 5, p. 1260-1266, 2011.

DEMO, P. Nova mídia e educação: incluir na sociedade do conhecimento. UNB, 2005.

EICHLER, M. L.; DEL PINO, J. C. Computadores em educação química: estrutura atômica e tabela periódica. Química Nova, n. 23, v.6, p. 835-840, 2000.

GALVÃO, T. F.; PEREIRA, M. G. Revisões sistemáticas da literatura: passos para sua elaboração. Rev. Epidemiol. Serv. Saúde, v. 23, n. 1, p. 183-184, 2014. 
GROS, B.; FORÉS, A. El uso de la geolocalización en educación secundaria para la mejora del aprendizaje situado/Using geolocation in secondary education to improve situated learning: analysis of two case studies. Revista Latinoamericana de Tecnología Educativa, v. 12, n. 2, p. 41-53, 2013.

HEIDEMANN, L. A.; ARAUJO, I. S.; VEIT, E. A. Atividades experimentais e atividades baseadas em simulações computacionais: quais os principais fatores que influenciam a decisão de professores de conduzir ou não essas práticas em suas aulas?. Revista Electrónica de Investigación en Educación en Ciencias, v. 9, n. 2, p. 42-57, 2014.

IGNÁCIO, A. C. O RPG Eletrônico no Ensino de Química: Uma Atividade Lúdica Aplicada ao Conhecimento de Tabela Periódica. 2013. 80 f. Dissertação (Mestrado) Universidade Tecnológica Federal do Paraná, Curitiba, 2013.

JESUS, S. M. C.; SILVA, M. D. F. Estudo das Funções Afins, Quadráticas e Equações Polinomiais com o auxílio do software Winplot no Ensino Médio. Revista Brasileira de Ensino de Ciência e Tecnologia, v. 8, n. 1, p. 70-91, 2015.

KITCHENHAM, B. Procedures for performing systematic reviews. Tech. Report TR/SE-0401 Keele, UK, Keele University, v. 33, p. 1-26, 2004.

LEÃO, M. B. C. Tecnologias na educação: uma abordagem crítica para uma atuação prática. Recife: EDUFRPE, 2011. 179 p.

LEITE, B. S. Aprendizagem tangencial no processo de ensino e aprendizagem de conceitos científicos: um estudo de caso. RENOTE, v. 14, n. 2, 2016.

LEITE, B. S. Tecnologias no ensino de química: teoria de prática na formação docente. Curitiba: Appris, 2015.

LEITE, B. S.; LEÃO, M. B. C. Contribuição da Web 2.0 como ferramenta de aprendizagem: um estudo de caso. Revista Brasileira de Ensino de Ciência e Tecnologia, v. 8, n. 4, p. 288-315, 2015.

MARTÍN, A. P. Flipped Learning: Aplicar el modelo de aprendizaje inverso. Madrid: Narcea, 2017.

OLIVEIRA, A. M. M.; MOREIRA, M. A. Um estudo exploratório para avaliar a dificuldade de problemas em ensino de física utilizando a teoria da carga cognitiva com o auxílio de uma hipermídia. Revista Brasileira de Ensino de Ciência e Tecnologia, v. 9, n. 1, p. 26-61, 2016.

PAULA, H. F. Fundamentos Pedagógicos para o Uso de Simulações e Laboratórios Virtuais no Ensino de Ciências. Revista Brasileira de Pesquisa em Educação em Ciências, v. 17, n. 1, p. 75-103, 2017.

PRETTO, N.L.; RICCIO, N. C. R. A formação continuada de professores universitários e as tecnologias digitais. Educar em Revista, n. 37, p. 153-169, 2010.

ROSA, M. P. A.; EICHLER, M. L.; CATELLI, F. "Quem me salva de ti?": representações docentes sobre a tecnologia digital. Ensaio Pesquisa em Educação em Ciências, v. 17, n. 1, p. 84-104, 2015.

SAMPAIO, R.F.; MANCINI, M. C. Estudos de Revisão Sistemática: um guia para síntese criteriosa da evidência científica. Revista brasileira de fisioterapia, v. 11, n. 1, p. 83-89, 2007.

SANTOS, M. P. Ensinando e Aprendendo Geometria Plana Através de Vídeo Educativo: Algumas Sugestões de Atividades Didáticas para Aulas de Matemática no Ensino Médio. Revista Brasileira de Ensino de Ciência e Tecnologia, v. 7, n. 3, p. 27 43, 2014.

SILVA, J. C.; BRITO, A. V. Levantamento bibliográfico das publicações acadêmicocientíficas sobre Análise de Redes Sociais aplicadas em E-Learning. RENOTE, v. 12, n. $1,2014$. 